\title{
Total protein, animal protein and physical activity in relation to muscle mass in middle-aged and older Americans
}

\author{
Martha Savaria Morris* and Paul F. Jacques \\ Nutritional Epidemiology Program, Jean Mayer USDA Human Nutrition Research Center on Aging, Tufts University, \\ 711 Washington Street, Room 901D, Boston, MA 02111, USA
}

(Submitted 7 December 2011 - Final revision received 8 June 2012 - Accepted 8 June 2012 - First published online 2 August 2012)

\begin{abstract}
Resistance training is recognised as a good strategy for retarding age-related declines in muscle mass and strength. Recent studies have also highlighted the potential value of protein intakes in excess of present recommendations. The roles that leisure-time physical activity and protein quality play in the preservation of skeletal muscle during ageing, and how such influences interact in free-living people are unclear. We sought to clarify these issues using data collected on 2425 participants aged $\geq 50$ years in the US National Health and Nutrition Examination Survey (2003-2006). We estimated subjects' usual intakes of total protein and beef from two $24 \mathrm{~h}$ diet recalls and computed the appendicular skeletal muscle mass index from anthropometric measures. Participants self-reported their physical activity levels. Analyses accounted for demographic factors and smoking. The association between muscle-strengthening activity and the appendicular skeletal muscle mass index varied with protein intake. Furthermore, among obese subjects with protein intakes $<70 \mathrm{~g} / \mathrm{d}$, those who performed such activities had a lower appendicular skeletal muscle mass index than those who were physically inactive. Protein intakes above the present recommendations were associated with benefits to obese subjects only. The appendicular skeletal muscle mass index of non-obese subjects who performed vigorous aerobic activities was consistently high; in obese subjects, it varied with protein intake. High-protein intake was associated with a modest increase in the appendicular skeletal muscle mass index in non-obese, physically inactive subjects. The present findings reinforce the idea that muscle-strengthening exercise preserves muscle when combined with adequate dietary protein. Vigorous aerobic activity may also help.
\end{abstract}

\section{Key words: Aerobic exercise: Ageing: Dietary proteins: Meat: Muscle atrophy: Sarcopenia}

An age-related loss of skeletal muscle is well known ${ }^{(1)}$, but research into the prevention of this problem began only recently - perhaps because it was thought to be inevitable ${ }^{(2)}$. In 1989, Rosenberg wrote the following in a plea for studies of the phenomenon, which he termed 'sarcopenia' (from the Greek roots sarx, for flesh, and penia, for loss) ${ }^{(3)}$ : 'No decline with age is more dramatic or potentially more functionally significant than the decline in lean body mass ${ }^{,(4)}$. Whether the functional significance of sarcopenia extends beyond its association with decreased muscle strength is currently a matter of debate ${ }^{(5)}$. However, decreasing muscle mass implies a dwindling reservoir of amino acids, which may threaten health by compromising protein synthesis in vital tissues and organs $^{(6)}$.

Muscle mass declines at a rate of $1-2 \%$ per year from age 50 years onwards ${ }^{(7)}$, but studies have shown that even very elderly people can build muscle through strength training $^{(8-13)}$. The more recent suggestion that high protein intake can enhance the effects of resistance training on muscle hypertrophy and strength ${ }^{(14)}$ is also considered relevant to the problem of sarcopenia ${ }^{(15)}$. Recent populationbased studies linked higher protein intakes among communitydwelling seniors to less muscle loss over time ${ }^{(16)}$ and a lower risk of frailty ${ }^{(17)}$. However, earlier investigations had reached the opposite conclusion ${ }^{(18,19)}$. Neither the independent effect of high protein intake on retarding muscle loss, nor the possibility of synergy between high protein intake and physical activity has yet been fully explored. Indeed, Koopman \& van Loon ${ }^{(13)}$ recently called for more research addressing the latter topic, citing scant evidence for augmentation of the effects of exercise training by dietary co-interventions. A related unanswered question concerns the effect of protein source on lean mass ${ }^{(16,17)}$. Houston et al. ${ }^{(16)}$ found significant associations between animal protein intake, but not vegetable protein intake, and longitudinal changes in lean body mass - a difference the authors attributed to the higher biological value of animal $v$. vegetable protein. This observation has not been confirmed, however, and findings of studies

Abbreviations: DXA, dual-energy X-ray absorptiometry; MEC, mobile examination center; NHANES, National Health and Nutrition Examination Survey. 
examining possible enhancement of resistive-training effects by meat consumption have been $\operatorname{mixed}^{(20,21)}$.

Glass \& Roubenoff ${ }^{(22)}$ encouraged the development of pharmacological treatment for muscle wasting because of the lack of known muscle-sparing strategies other than resistive-exercise training, which cannot be practically applied to large populations. However, little is known about the muscle-sparing potential of the kinds of physical activity routinely performed by the general population of ageing people. The goal of the present investigation was to address some of these unanswered questions by performing cross-sectional analyses using data collected on participants aged $\geq 50$ years in the National Health and Nutrition Examination Survey (NHANES, 2003-2006).

\section{Experimental methods}

\section{Study population}

The subjects in the present investigation were NHANES 2003-2006 participants aged $\geq 50$ years. The NHANES is a survey research programme conducted by the US National Center for Health Statistics to assess the health and nutritional status of adults and children in the USA, and to track changes over time. Findings from the survey are used to set standards, to develop public health policy, to determine the prevalence of major diseases and risk factors for diseases and to evaluate associations between nutritional status and health promotion and disease prevention.

The NHANES is currently implemented as a continuous annual survey and uses a complex multi-stage probability design to select a representative sample of the non-institutionalised, civilian US population. To increase the precision of estimates derived from the survey, adolescents, the elderly, Mexican Americans and blacks are oversampled. The protocols for conducting the NHANES were approved by the institutional review board of the National Center for Health Statistics, Centers for Disease Control and Prevention, and informed consent was obtained from all participants ${ }^{(23)}$. Trained interviewers used a computer-assisted personal interview system to query participants at their homes. The participants were also asked to report to a mobile examination center (MEC) after a 6 or $9 \mathrm{~h}$ fast to provide further interview data and undergo a physical examination that included phlebotomy.

At the MEC, subjects provided dietary data in a $24 \mathrm{~h}$ diet recall. All of those who did so were eligible for a second $24 \mathrm{~h}$ diet recall conducted by telephone, 3-10 d after the MEC examination. During the years 2003-2006, subjects who completed both $24 \mathrm{~h}$ recalls were sent a FFQ to complete and return. The FFQ, which was non-quantitative, was designed to assess usual dietary intake and queried subjects about their frequency of consuming foods and food groups during the previous 12 months.

In 2003-2004, body composition was assessed at the MEC by using dual-energy X-ray absorptiometry (DXA). The DXA scans provided bone and soft tissue measurements for the total body, both arms and legs, the trunk and the head. In all four study years, anthropometric data that could be used to estimate body composition were collected. The relevant measures included triceps and sub-scapular skinfold thicknesses and the circumferences of the upper arm, thigh and calf.

Questionnaires administered at the MEC also collected medical and smoking histories and physical activity data.

In the present study, we performed secondary statistical analyses on data downloaded from the NHANES website ${ }^{(24)}$. The present project was certified to be exempted from approval by the Tufts University Health Sciences Campus Institutional Review Board (no. 9145). Consistent with NHANES analytic guidelines for obtaining precise estimates ${ }^{(25)}$, we combined data from two surveys (i.e. 2003-2004 and 2005-2006) into a single data set.

The NHANES 2003-2006 participants aged $\geq 50$ years and numbered 4724 . However, $10.3 \%$ of these subjects did not participate in the MEC examination. Of the 4236 MEC-examined subjects, dietary, anthropometric and physical activity data needed for analyses were collected for 2425 participants.

\section{Estimation of the appendicular skeletal muscle mass index}

There is no generally agreed upon definition of sarcopenia $^{(26,27)}$. Furthermore, we would only expect to find sarcopenia among the very old, who were not numerous in the present data set. However, evidence suggests that the tendency towards sarcopenia begins in the age range of the present study subjects ${ }^{(7,28)}$. Consequently, our outcome measure in multivariate models was the appendicular skeletal muscle mass index (i.e. estimated appendicular skeletal muscle mass divided by height in $\left.\mathrm{m}^{2}\right)^{(27)}$ as a continuous term.

Participants in the 2003-2004 survey underwent whole-body DXA scans, but participants in the 2005-2006 survey did not. Of the 2425 subjects eligible to test the present hypotheses from the two surveys, 557 men and 625 women had undergone DXA scanning. We combined the DXA results of those subjects with those of an additional 290 men and 167 women for whom dietary, smoking and/or physical activity data were lacking to estimate the appendicular skeletal muscle mass from anthropometric measures. We defined the 'gold-standard' estimate of the appendicular skeletal muscle mass as the sum of the four DXA-derived estimates of lean limb mass excluding the bone mineral content. To derive the equation for each sex, we created a model for the DXA-estimated appendicular skeletal muscle mass with terms for age, height and race-ethnicity (i.e. non-Hispanic white, non-Hispanic black, Mexican American, other Hispanic and other race) forced in. We then added terms for each of the following anthropometric measures one at a time: calf circumference, arm circumference, thigh circumference, triceps skinfold thickness and sub-scapular skinfold thickness. For both sexes, the model with terms for age, height, race-ethnicity and calf circumference explained $>70 \%$ of the variation in the appendicular skeletal mass. For men, adding terms for arm circumference and triceps skinfold thickness resulted in a model that explained $86 \%$ of the variation (see Appendix). The final model that we arrived at for women explained $79 \%$ of the variation and included terms for arm and thigh circumference. 


\section{Estimation of usual intake of beef and total protein}

Beef. The US Environmental Protection Agency has estimated the day 1 and day 2 beef intakes of the participants in the NHANES 2003-2006 surveys. This was part of a larger effort to estimate the subjects' intakes of a variety of agricultural commodities ${ }^{(29)}$. The estimates were derived from the gram amounts of foods consumed and preparation methods specified in the $24 \mathrm{~h}$ recall and standard recipes for the approximately 7000 food codes to which all reported foods were assigned. The codes are for single- and mixed-meal foods. The EPA's estimates of meat intakes exclude portions of the food not eaten (e.g. bone and fat, if the subject reported eating only the lean portion).

Beef is an episodically consumed food. That is, few people who consume beef consume it every day. For this reason, even two $24 \mathrm{~h}$ recalls may not capture any beef when beef is consumed relatively frequently. As Dwyer put it simply, 'Two $24 \mathrm{~h}$ recalls, if not supplemented, overestimate the number of non-consumers ${ }^{,(30)}$. To minimise this problem, we followed the NHANES recommendation to use macros created by the National Cancer Institute ${ }^{(31)}$. Estimation of an individual's usual intake of an episodically consumed food by these macros proceeds by several steps accomplished by using multivariate modelling procedures. The general premise for the method is that a person's usual intake of such a food is given by the product of the probability of consuming the food on any given day and the amount consumed on a consumption day. The first step was prediction of each subject's probability of consuming beef. This was accomplished by using a logistic regression procedure. The binary outcome for the model was ' 1 ' if some beef was reported on a $24 \mathrm{~h}$ recall and ' 0 ' if no beef was reported. The second multivariate modelling step was used to predict the consumption-day amount of the food from the data provided in the $24 \mathrm{~h}$ recalls and the same covariates as used in the logistic regression model. The covariates for both models were age, sex, race-ethnicity, attained educational level and frequency of beef consumption from the FFQ. Each subject's linear predictors were estimated for both models, and a final estimate of usual beef intake was obtained by evaluating a ratio of integrals integrated over the person-specific effects using adaptive Gaussian quadrature. Consistent with our interest in identifying benefits specifically associated with consuming beef, we also estimated each subject's total pork intake for comparison purposes.

Protein. For all macronutrients, many micronutrients and energy, the NHANES provides the total amount consumed by each subject on the day of each $24 \mathrm{~h}$ recall. To estimate usual protein and energy intake, we input the values for protein and energy from the separate recalls into the National Cancer Institute macro programmes - skipping the probability part of the model, as everyone consumes some energy-providing food every day, and protein is consumed by everyone either every day or nearly every day. Covariates in the multivariate models were age, sex, race-ethnicity and attained educational level.

\section{Physical activity assessment}

One of the MEC-administered questionnaires was concerned with physical activity ${ }^{(32)}$. To classify subjects in the present investigation according to their physical activity status, we used their responses to three questions relating to the previous $30 \mathrm{~d}$. For vigorous aerobic activity, the subjects were asked whether they had performed any vigorous activities for at least $10 \mathrm{~min}$ that caused heavy sweating or large increases in breathing or heart rate. Examples offered to the subjects were running, lap swimming, aerobics classes and fast bicycling. For moderate aerobic activity, the subjects were asked whether they had performed any moderate activities for at least $10 \mathrm{~min}$ that caused only light sweating or a slight to moderate increase in breathing or heart rate. Examples offered to the subjects were brisk walking, bicycling for pleasure, golf and dancing. For muscle-strengthening activities, the subjects were asked whether they had performed any physical activities specifically designed to strengthen their muscles. Examples offered to the subjects were weight lifting, push-ups and sit-ups. For each type of physical activity, we used a two-level term in the multivariate statistical modelling - coding the subjects ' 1 ' for an affirmative response to the question and ' 2 ' for a negative response.

\section{Statistical methods}

All statistical analyses were carried out using SUDAAN release 10.0.1 (Research Triangle Institute) with appropriate sampling weights, pseudo primary sampling units and stratification variables from the data set to account for the survey's complex sampling design ${ }^{(25)}$. We assessed statistical significance at $P<0.05$ using two-sided tests.

Using means (95\% CI) and proportions, we first described the study population according to various subject characteristics.

The main data analyses in the present study involved determining how physical activity status and usual intakes of beef and total protein were related to the appendicular skeletal muscle mass index. To investigate the possible synergy between physical activity and the dietary factors, we tested the interactions between physical activity status and the dietary factors in relation to the appendicular skeletal muscle mass index.

Physical activity and usual intakes of beef and total protein in relation to the appendicular skeletal muscle mass index. To relate physical activity type and the dietary factors to the appendicular skeletal muscle mass index, we used a multiple linear regression model with our estimate of the appendicular skeletal muscle mass index as the continuous outcome variable and terms for the following characteristics as predictors: age (continuous), sex, race-ethnicity, current smoking status (yes/no), obesity status (BMI >30 v. $\leq 30$ ), vigorous aerobic activity (yes/no), moderate aerobic activity (yes/no), musclestrengthening exercises (yes/no) and usual beef intake (continuous) or usual total protein intake (continuous). We performed the analysis relating protein intake to the appendicular skeletal muscle mass index, with the usual 
intakes estimated using the National Cancer Institute method, and also after adjusting those estimates for energy intake by the residuals method ${ }^{(33)}$.

To graphically illustrate the dose-response relationship between usual dietary intakes and the appendicular skeletal muscle mass index in subjects stratified by physical activity status, we obtained and plotted predicted values of the index for usual weekly beef intakes and usual daily protein intakes that corresponded to the medians of six equal-sized groups. Because of differences between obese and nonobese subjects in the appendicular skeletal muscle mass index and some significant differences in the trends in the index with increasing dietary intakes, the graphs are presented separately for obese and non-obese subjects.

We also considered how meeting or exceeding ( $v$. not meeting) the present RDA for protein of $0.8 \mathrm{~g} / \mathrm{kg}$ per $\mathrm{d}$ was related to the appendicular skeletal muscle mass index. For this analysis, we substituted a three-level term for the estimate of usual protein intake in the multivariate model. The three categories comprised subjects with intakes per $\mathrm{kg}<0.8$ (not meeting the RDA, $n$ 468), from 0.8 to $1.0 \mathrm{~g}$ (meeting the RDA, $n$ 806) and $>1.0 \mathrm{~g}$ (exceeding the RDA, $n 1,151$ ).

\section{Results}

\section{Subject characteristics}

The mean age of the subjects was 63 years (Table 1). The distribution of the subjects according to sex and race-ethnicity reflected the survey's goal of representing the non-institutionalised, civilian US population. A total of $15 \%$ of the subjects smoked cigarettes and over one-quarter of them were obese. A total of $67 \%$ reported engaging in some form of physical activity. Moderate aerobic activity was reported by $58 \%$ of the subjects, and about half that many said they had engaged in vigorous aerobic activity or muscle-strengthening exercises. The subjects consumed about $260 \mathrm{~g}$ /week of beef, on average, which reflected $318 \mathrm{~g} /$ week for men and $210 \mathrm{~g} /$ week for women. The corresponding annual averages of $16 \mathrm{~kg} /$ year for men and $11 \mathrm{~kg} /$ year for women are much lower than the 30 and $20 \mathrm{~kg} /$ year estimated for senior men and women, respectively, by the United States Department of Agriculture based on Continuing Survey of Food Intakes by Individuals data collected in the late $1990 \mathrm{~s}^{(34)}$

In all, $9 \%$ of the non-obese subjects failed to meet the present RDA for protein of $0.8 \mathrm{~g} / \mathrm{kg}$ per $\mathrm{d}$, and $61 \%$ had intakes $>1.0 \mathrm{~g} / \mathrm{kg}$ per $\mathrm{d}$. The corresponding percentages for obese subjects were 53 and 9\%. After multivariate adjustment for age, sex and race-ethnicity, mean estimated appendicular skeletal muscle mass was $19 \cdot 1 \mathrm{~kg}$ for the non-obese men and $15.4 \mathrm{~kg}$ for the non-obese women. Estimated appendicular skeletal muscle mass was much higher for obese men and women $(P<0.001)-28.6$ and $24.4 \mathrm{~kg}$, respectively. Beef intakes were significantly correlated with total protein intakes $(r 0 \cdot 19, P<0 \cdot 0001)$.

\section{Subject characteristics and the appendicular skeletal muscle mass index}

Higher age, female sex, Mexican American race-ethnicity and smoking were related to a lower appendicular skeletal muscle mass index, while non-Hispanic black race-ethnicity, obesity and usual intakes of beef and total protein were positively related to this outcome (Table 2). Neither moderate aerobic activity nor muscle-strengthening activity was related to the appendicular skeletal muscle mass index. However, consideration of possible interactions between usual intakes of beef and total protein and muscle-strengthening exercises suggested that the association between performing this type of physical activity and the appendicular skeletal

Table 1. Characteristics of 2425 participants in the National Health and Nutrition Examination Survey (2003-2006) aged $\geq 50$ years from whom dietary and anthropometric data were collected

(Mean values, ranges, percentages, number of participants and $95 \%$ confidence intervals)

\begin{tabular}{|c|c|c|c|}
\hline Characteristic & $n$ & Mean or percentage* & $95 \% \mathrm{Cl}^{\star}$ \\
\hline Age (years) $\dagger$ & & $63 \cdot 0$ & $62 \cdot 3,63 \cdot 8$ \\
\hline Range & & $50-85$ & \\
\hline \multicolumn{4}{|l|}{$\operatorname{Sex}(\%)$} \\
\hline Female & 1168 & $55 \cdot 6$ & $53 \cdot 8,57 \cdot 4$ \\
\hline \multicolumn{4}{|l|}{ Race-ethnicity (\%) } \\
\hline Non-Hispanic white & 1632 & $84 \cdot 7$ & $80 \cdot 4,88 \cdot 1$ \\
\hline Non-Hispanic black & 349 & $6 \cdot 3$ & $4 \cdot 6,8 \cdot 6$ \\
\hline Mexican American & 324 & 2.9 & $1 \cdot 8,4.6$ \\
\hline Smoker (\%) & 338 & 14.9 & $13 \cdot 1,16 \cdot 9$ \\
\hline Obese $\left(\mathrm{BMI}>30 \mathrm{~kg} / \mathrm{m}^{2}\right)(\%)$ & 636 & $26 \cdot 6$ & $23 \cdot 7,29 \cdot 7$ \\
\hline Moderate physical activity (\%) & 1269 & $58 \cdot 2$ & $55 \cdot 3,60 \cdot 9$ \\
\hline Muscle strengthening (\%) & 548 & $25 \cdot 4$ & $22 \cdot 7,28 \cdot 4$ \\
\hline Vigorous aerobic activity (\%) & 455 & 24.5 & $22 \cdot 0,27 \cdot 2$ \\
\hline Beef intake (g/week) & & 257.9 & $251 \cdot 0,264 \cdot 8$ \\
\hline Range & & $10 \cdot 3-646 \cdot 7$ & \\
\hline Total protein intake $(\mathrm{g} / \mathrm{d}) \ddagger$ & & $76 \cdot 6$ & $76 \cdot 2,77 \cdot 1$ \\
\hline Range & & $25 \cdot 3-122 \cdot 2$ & \\
\hline
\end{tabular}

*Values in this column are weighted to take into account the survey's complex sampling design. t The highest age recorded for subjects aged $>85$ years was 85 years.

$\ddagger$ Adjusted for energy intake by the residuals method. 
Table 2. Associations between characteristics of 2425 participants in the National Health and Nutrition Examination Survey (2003-2006) aged $\geq 50$ years and the appendicular skeletal muscle mass index

\begin{tabular}{|c|c|c|}
\hline Characteristic & $\beta^{*}$ & $P^{*}$ \\
\hline Age (years) & -0.02 & $<0.001$ \\
\hline \multicolumn{3}{|l|}{ Sex } \\
\hline Female & -1.99 & $<0.001$ \\
\hline \multicolumn{3}{|l|}{ Race-ethnicity } \\
\hline Non-Hispanic white & Referent & Referent \\
\hline Non-Hispanic black & 0.76 & $<0.001$ \\
\hline Mexican American & -0.16 & 0.015 \\
\hline Smoker & -0.32 & $<0.001$ \\
\hline $\mathrm{BMI}>30 \mathrm{~kg} / \mathrm{m}^{2}$ & 1.5 & $<0.001$ \\
\hline Moderate aerobic activity & 0.02 & 0.677 \\
\hline Muscle-strengthening activity & -0.04 & 0.33 \\
\hline \multicolumn{3}{|l|}{ Vigorous aerobic activity† } \\
\hline $\mathrm{BMI} \leq 30 \mathrm{~kg} / \mathrm{m}^{2}$ & $0 \cdot 12$ & 0.015 \\
\hline $\mathrm{BMI}>30 \mathrm{~kg} / \mathrm{m}^{2}$ & -0.09 & 0.338 \\
\hline Usual beef intake & 0.03 & 0.005 \\
\hline Usual total protein intake & 0.05 & 0.002 \\
\hline \multicolumn{3}{|c|}{$\begin{array}{l}\text { *Multivariate-adjusted association between the characteristic and the skeletal } \\
\left.\text { muscle mass index (estimated skeletal muscle mass }(\mathrm{kg}) / \text { height }\left(\mathrm{m}^{2}\right)\right) \text {. Estimates } \\
\text { are adjusted for all of the other listed factors except usual intakes of beef and } \\
\text { total protein }\end{array}$} \\
\hline
\end{tabular}

muscle mass index varied with usual intakes of beef $\left(P_{\text {interaction }}=0.028\right)$ and total protein $\left(P_{\text {interaction }}=0.047\right)$. A positive association between vigorous aerobic activity and the appendicular skeletal muscle mass index was restricted to the non-obese subjects. However, consideration of possible interactions between usual intakes of beef and total protein and this type of physical activity suggested that the association in obese subjects between performing vigorous aerobic activities and the appendicular skeletal muscle mass index varied with usual intakes of beef $\left(P_{\text {interaction }}=0.023\right)$ and total protein $\left(P_{\text {interaction }}=0 \cdot 018\right)$.

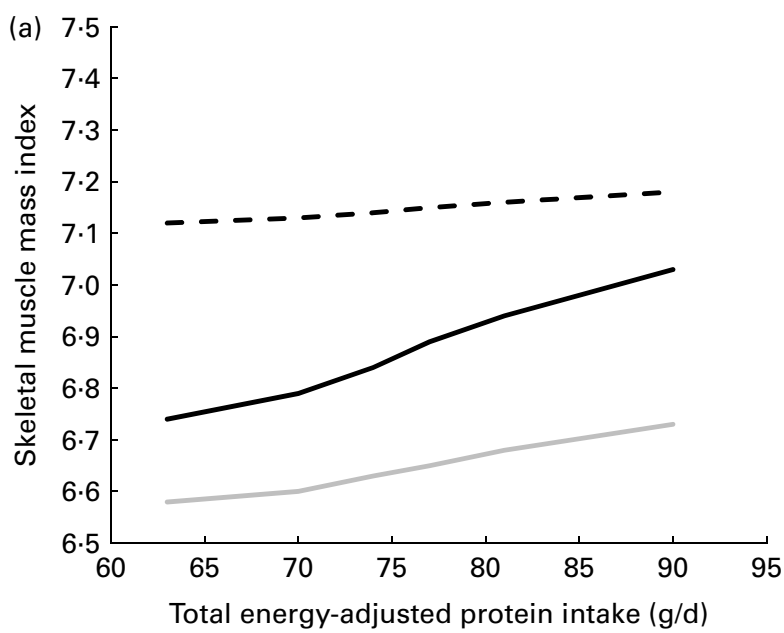

Interaction between physical activity and usual intakes of beef and total protein in relation to the appendicular skeletal muscle mass index

Results for subjects who performed moderate aerobic activities but no vigorous aerobic activities or muscle-strengthening exercises were nearly identical to the results for subjects who did not perform any of the three types of activities. We therefore combined the two groups into a single referent category. Results for pork intake were very similar to results for beef intake and are not shown.

Non-obese subjects. Among the non-obese subjects, engaging in vigorous aerobic activity was associated with a high appendicular skeletal muscle mass index, regardless of total protein intake (Fig. 1(a)), but the mean appendicular skeletal muscle mass index of these subjects was modestly, but significantly, increased in association with higher usual beef intakes (Fig. 1(b)). However, dietary intake of protein or beef appeared to be more important to non-obese subjects who performed muscle-strengthening exercises, in that the mean appendicular skeletal muscle mass index for those subjects only approached that of subjects who performed vigorous aerobic activities when muscle-strengthening was combined with a diet high in total protein or beef. Relationships between intakes of both beef and total protein and the appendicular skeletal muscle mass index in nonobese subjects who performed muscle-strengthening exercises remained strong and significant when terms for both dietary factors were included in the same model (protein, $10 \mathrm{~g} / \mathrm{d}$ increase, $\beta=0.12, P=0.012$; beef, $100 \mathrm{~g}$ / week increase, $\beta=0.12, P=0.015)$.

Among non-obese subjects who performed neither vigorous aerobic activities, nor muscle-strengthening exercises, a small but significant advantage to the appendicular skeletal muscle mass was associated with higher total protein intakes.

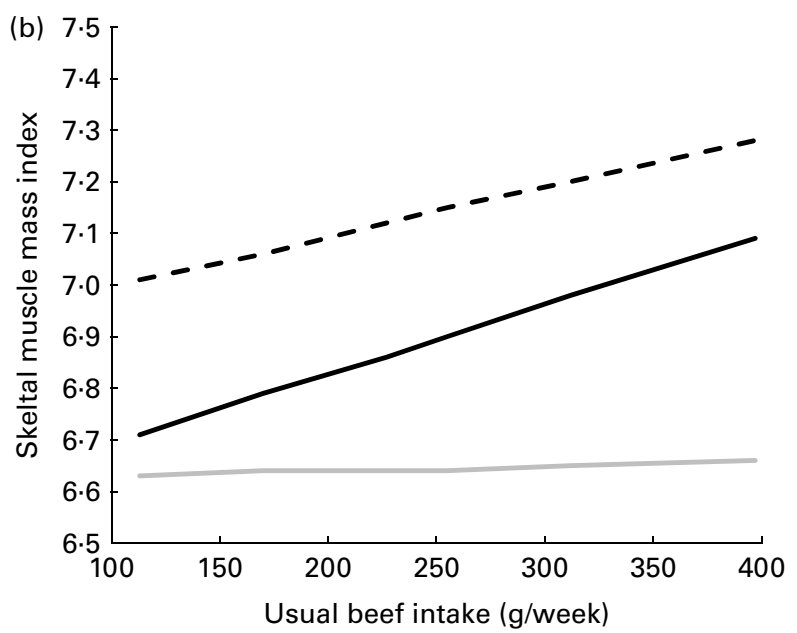

Fig. 1. Increase in the appendicular skeletal muscle mass index (i.e. estimated appendicular skeletal muscle mass $(\mathrm{kg}) / \mathrm{height}\left(\mathrm{m}^{2}\right)$ ) $\mathrm{with}$ increasing usual intake of (a) total protein and (b) beef, by self-reported physical activity status, among 1789 non-obese participants in the National Health and Nutrition Examination Survey aged $\geq 50$ years. Results are adjusted for age, sex, race-ethnicity, smoking status and other forms of physical activity. $\beta$-Coefficients give the increase per $10 \mathrm{~g}$ increase in daily protein intake and $100 \mathrm{~g}$ increase in weekly beef intake. (a) -- , Vigorous aerobic $(\beta=0.02, P=0.551)$; - , muscle-strengthening $(\beta=0.12, P=0.012) ;-$, moderate aerobic or none $(\beta=0.06, P=0.022)$. (b) -- , Vigorous aerobic $(\beta=0.10, P=0.038) ;-$, muscle-strengthening $(\beta=0.13$, $P=0.006) ;-$, moderate aerobic or none $(\beta=0.01, P=0.804)$. 

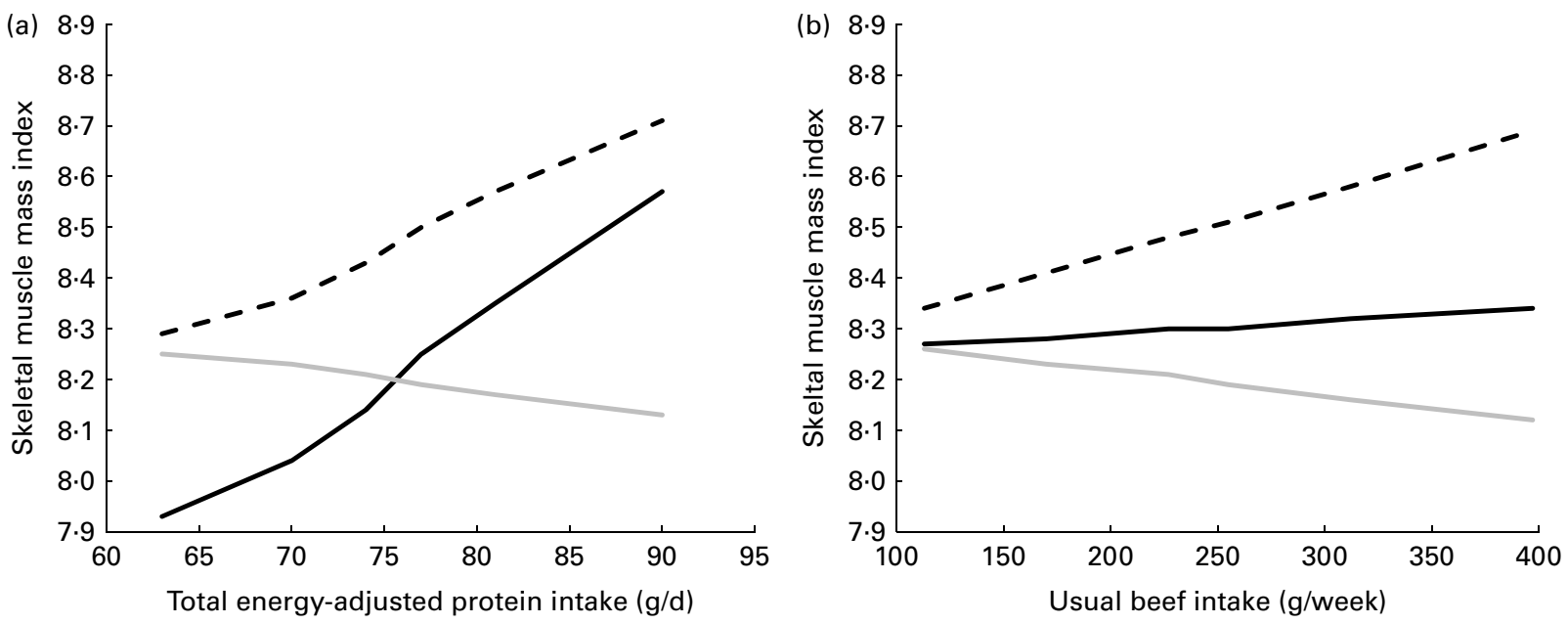

Fig. 2. Increase in the appendicular skeletal muscle mass index (i.e. estimated appendicular skeletal muscle mass $(\mathrm{kg}) / \mathrm{height}\left(\mathrm{m}^{2}\right)$ ) with increasing usual intake of (a) total protein and (b) beef, by self-reported physical activity status, among 636 obese participants in the National Health and Nutrition Examination Survey aged $\geq 50$ years. Results are adjusted for age, sex, race-ethnicity, smoking status and other forms of physical activity. $\beta$-Coefficients give the increase per $10 \mathrm{~g}$ increase in daily protein intake and $100 \mathrm{~g}$ increase in weekly beef intake. (a) -- , Vigorous aerobic $(\beta=0.17, P=0.019) ;-$, muscle-strengthening $(\beta=0.26$, $P<0.001)$; — moderate aerobic or none $(\beta=-0.05, P=0.428)$. (b) -- , Vigorous aerobic $(\beta=0.12, P=0.183)$; - , muscle-strengthening $(\beta=0.02$, $P=0.778) ;-$, moderate aerobic or none $(\beta=-0.05, P=0.371)$.

However, in comparison to the physically active subjects, the physically inactive subjects had low appendicular skeletal muscle mass, regardless of their protein intakes.

Obese subjects. Associations between total protein intake and the appendicular skeletal muscle mass index were stronger in physically active obese subjects than they were in physically active non-obese subjects (vigorous aerobic activity, $\quad P_{\text {interaction }}=0.07 ;$ muscle-strengthening exercise, $\left.P_{\text {interaction }}=0.049\right)$. Most importantly, among obese subjects who consumed $<70 \mathrm{~g} / \mathrm{d}$ of protein, those who performed muscle-strengthening exercises had a significantly lower mean appendicular skeletal muscle mass index than physically inactive subjects $(\beta=-0 \cdot 48, P=0 \cdot 013$; Fig. $2(\mathrm{a}))$. Although no such disadvantage was associated with vigorous aerobic activity at any protein-intake level, no advantage was associated with performing vigorous aerobic activities unless the activity was combined with a high-protein diet.

Beef intake was positively associated with the appendicular skeletal muscle mass index only in those obese subjects who performed vigorous aerobic activities (Fig. 2(b)). When terms for usual beef intake and total protein intake were included in the same model, the $\beta$-coefficients relating a $10 \mathrm{~g} / \mathrm{d}$ increment in protein intake and a $100 \mathrm{~g} /$ week increment in usual beef intake to the appendicular skeletal muscle mass index changed from $0 \cdot 17(P=0 \cdot 019)$ to $0 \cdot 14(P=0 \cdot 052)$, and from $0 \cdot 12(P=0 \cdot 183)$ to $0 \cdot 16(P=0 \cdot 102)$, respectively.

Protein recommendations. Results of the analysis investigating the association between degree of adherence to the present RDA for protein and the appendicular skeletal muscle mass index in subjects stratified by obesity and physical activity status mirrored those displayed in Figs. 1(a) and 2(a) shown earlier. Specifically, among non-obese subjects who performed vigorous aerobic activities, the appendicular skeletal muscle mass index was high regardless of whether the RDA was met, not met or exceeded (Fig. 3(a)). Among the other two subgroups of non-obese subjects, however, those who met the RDA had a significantly higher mean appendicular skeletal muscle mass index than those who did not. However, regardless of physical activity status, no advantage was associated with far exceeding $v$. meeting or slightly exceeding the RDA.

Among the obese subjects, some advantage was associated with exceeding the RDA for all subgroups, and the only subgroup in which just meeting the RDA appeared advantageous comprised subjects who performed muscle-strengthening exercises. It is also important to emphasise that, regardless of obesity status, subjects who performed muscle-strengthening exercises had greater appendicular skeletal muscle mass than physically inactive subjects only if they met or exceeded the RDA for protein.

\section{Discussion}

Sarcopenia occurs frequently in old age ${ }^{(35)}$. That all elderly people do not become sarcopenic suggests that the condition is preventable. Muscle building occurs by increased protein synthesis ${ }^{(36)}$. Consequently, protein intake should be important to supply amino acids for this process, and studies have borne this out ${ }^{(2)}$. The present findings are consistent with this idea because we found that higher intakes of beef, a good source of protein, and higher total protein intakes were associated with a higher appendicular skeletal muscle mass index. The present results in this regard echo those of Houston et al. ${ }^{(16)}$, who reported less loss of appendicular skeletal muscle by 2066 community-dwelling seniors over 3 years of follow-up in association with higher intakes of total and animal protein. The association between protein intake and the appendicular skeletal muscle mass and its preservation over time may translate to functional benefits, because Beasley et $a l^{(17)}$ recently found that higher protein intakes were 

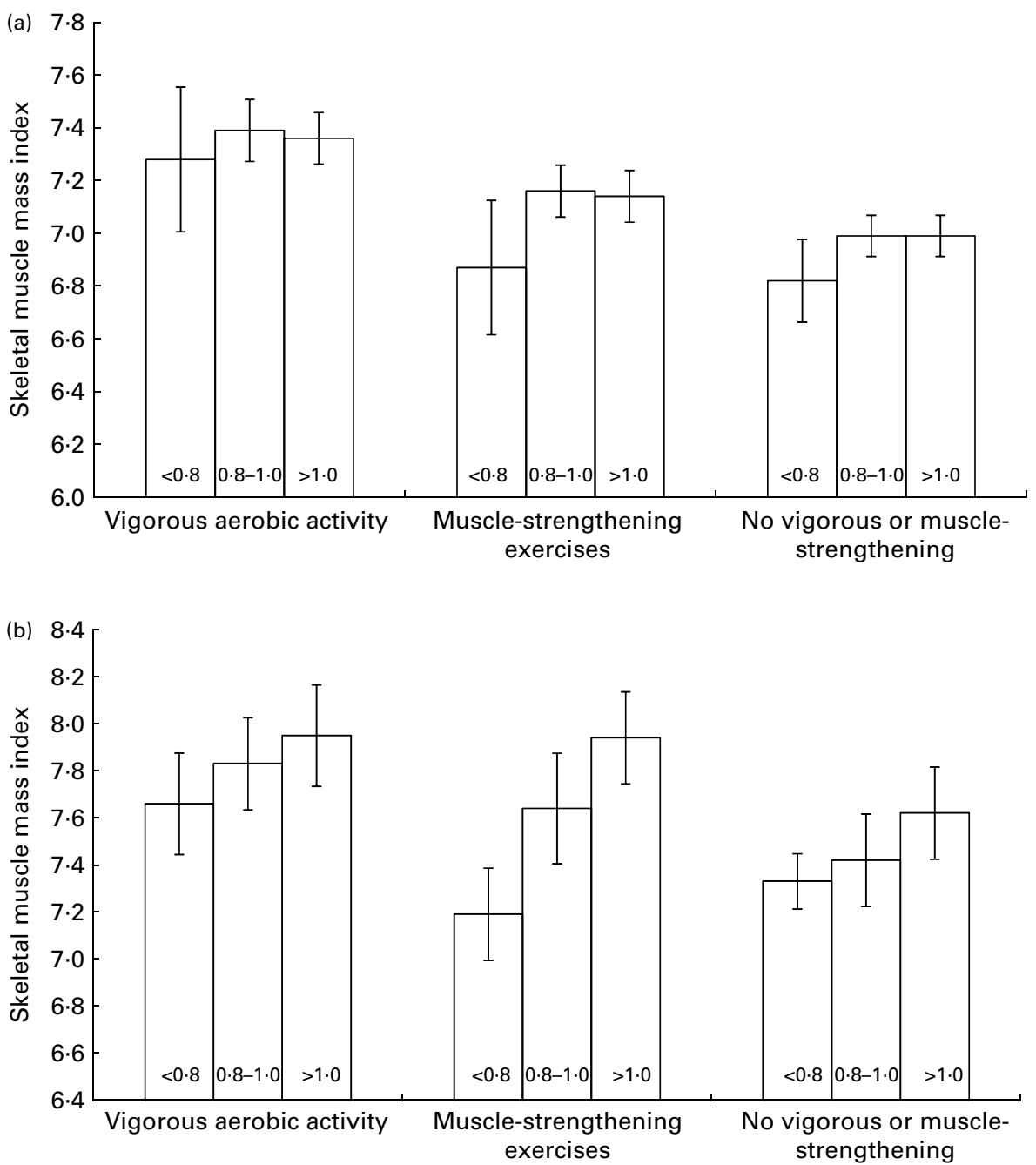

Fig. 3. Relationship between meeting or exceeding ( $v$. not meeting) the RDA for protein intake of $0.8 \mathrm{~g} / \mathrm{kg}$ per $\mathrm{d}$ and the appendicular skeletal muscle mass index (i.e. estimated appendicular skeletal muscle mass $(\mathrm{kg}) /$ height $(\mathrm{m})^{2}$ ), by self-reported physical activity status, among 1789 non-obese and 636 obese participants in the National Health and Nutrition Examination Survey aged $\geq 50$ years. Results are adjusted for age, sex, race-ethnicity, smoking status, other forms of physical activity and body weight.

associated with a reduced incidence of frailty over time among $>24000$ participants aged 65-79 years in the Women's Health Initiative. Both longitudinal studies found that significant benefits accrued only to study participants who consumed more than the present RDA for protein of $0.8 \mathrm{~g} / \mathrm{kg}$ per $\mathrm{d}$. The present results for obese subjects were consistent with this finding. However, exceeding the RDA was not associated with additional benefits to skeletal muscle mass among non-obese subjects beyond those associated with just meeting the RDA

Results of many previous studies supported the view that muscle protein synthesis is responsive to strength or resistance training in young and old alike ${ }^{(2)}$. Furthermore, Peterson et $a{ }^{(37)}$ recently concluded from a systematic review and meta-analysis of forty-nine studies that resistance exercise is effective for eliciting gains in lean body mass among ageing adults. The present study did not suggest overall benefits to skeletal muscle mass of performing muscle-strengthening activities. However, further analyses suggested that the lack of an overall association resulted from summarising results over all the levels of protein intake. Specifically, benefits to skeletal muscle mass of performing muscle-strengthening exercises applied only to those subjects with high-protein intakes. In fact, obese subjects who combined musclestrengthening exercises with low-protein intakes, or intakes below the RDA, had a lower mean appendicular skeletal muscle mass index than physically inactive obese subjects. These findings, although specific to the obese NHANES participants, are relevant to the controversy over whether physically active people have an increased requirement for protein $^{(38,39)}$, and may be consistent with Campbell et al.'s ${ }^{(21)}$ observation of a loss of whole-body fat-free mass and muscle mass in men who consumed the RDA for protein during a 12 week period of resistance training.

Results of intervention studies have been mixed concerning the role of protein quality in preserving muscle mass during resistance training ${ }^{(20,21,40)}$. The present findings were also mixed on this point. Specifically, higher usual beef intakes were positively related to the appendicular skeletal muscle mass index among the non-obese subjects who performed 
muscle-strengthening exercises, but not among the obese subjects who performed such activities.

Perhaps due to the prominent role that strength training plays in body building, few studies have attempted to evaluate the benefits of aerobic activity. However, the hypothesis that it might build or preserve skeletal muscle has been raised ${ }^{(41)}$. A small study that compared male running-club members to comparably aged sedentary men found that, even among those aged $\geq 60$ years, limb muscle mass was higher in the runners than in their sedentary counterparts ${ }^{(42)}$. The NHANES data on the specific aerobic activities performed revealed that few of the subjects who performed vigorous aerobic activities were runners. Nevertheless, we found that performing vigorous aerobic activities was positively associated with the appendicular skeletal muscle mass index in all subjects combined, in the non-obese subjects and in the obese subjects with high-protein or beef intakes. Thus, the present results may suggest that lower-impact aerobic activities such as fast bicycling and lap swimming are of value in preserving skeletal muscle as people age.

Although total protein intake appeared to be irrelevant to the benefits associated with performing vigorous aerobic activities among the non-obese subjects, results for both obese and non-obese subjects generated the hypothesis that high-quality protein foods like beef might enhance the benefits of that type of activity. An increased need for high-quality protein among older people who engage in vigorous aerobic activities could derive from the oxidation of amino acids, which results from such activities ${ }^{(12)}$, and the particular importance of essential amino acids to muscle anabolism in the elderly ${ }^{(13)}$

We had no prior hypotheses regarding differences between obese and non-obese people. Therefore, the present findings for the obese subgroup warrant cautious interpretation. On the other hand, the present results should inspire future research into possible interactions between diet and physical activity in this large segment of the population. A regimen of muscle-strengthening exercises might seem like an appropriate prescription for combatting sarcopenic obesity ${ }^{(43)}$. However, the present study raises the possibility that such a regimen might do more harm than good unless the RDA for protein is at least met. The present study also showed that meeting the RDA was rare for obese people and highlighted possible benefits of exceeding the RDA to them.

We have no immediate explanation for the variation in the present findings with obesity status. However, the differences we found should inform the controversy over the adequacy of the present RDA for protein, both with and without physical activity. Obesity status may be just one characteristic upon which the question of adequacy depends.

Among the major strengths of the present study was the effort invested by the NHANES in collecting the data needed to estimate usual long-term intakes of episodically consumed foods like beef. Furthermore, the oversampling of the elderly allowed for precise estimation of the strength of associations and provided the statistical power to detect them. Study limitations included the cross-sectional design and the restricted availability of the DXA measures upon which estimates of the appendicular skeletal muscle mass were based. Although the physical activity assessment by self-report was also a weakness, we were able to identify subgroups that appeared to be relatively more protected from a tendency towards sarcopenia than the rest of the sample. Furthermore, the use of a general-population sample and the observational study design allowed us to consider the effects of the kinds of physical activity that older people voluntarily participate in and can sustain. As a result, we were able to elucidate benefits of a type of activity that people begin early in life and continue, or adopt late in life for expected benefits other than sarcopenia prevention. Baumgartner et al. ${ }^{(19)}$ previously demonstrated strong correlations between physical activity and muscle mass among 301 elderly volunteers in New Mexico. That study was unable to attribute the effect to any particular type of exercise. However, as none of the subjects were engaged in formal exercise programmes, the activities performed by those with high physical activity scores likely resembled the vigorous aerobic activities reported by the present study subjects. These findings thus seem to conflict with Evans's ${ }^{(12)}$ conclusion from a comprehensive review that, 'While both aerobic and strength conditioning are highly recommended, only strength training can stop or reverse sarcopenia'.

Besides confirming or refuting the present findings, future studies should focus on identifying the specific types and amounts of physical activity that can build or preserve skeletal muscle in the elderly. They should also seek to clarify the types and amounts of protein needed under varying conditions in middle and later life. As cross-sectional studies cannot prove cause-and-effect relationships, specific recommendations should be based on results of diet-exercise intervention studies.

\section{Conclusion}

The findings from the present cross-sectional, populationbased study generate the hypothesis that engaging in vigorous aerobic activity can help to preserve skeletal muscle during ageing - particularly if accompanied by a diet rich in highquality protein. The present results also corroborate the idea that muscle-strengthening exercise is associated with benefits to muscle mass if supported by adequate dietary protein. Finally, protein intakes that exceeded the present RDA were associated with benefits to obese subjects only.

\section{Acknowledgements}

The present project was funded in part by The Beef Checkoff through the National Cattlemen's Beef Association and by the United States Department of Agriculture's Agricultural Research Service under agreement no. 58-1950-7-707. The authors have no conflicts of interest to declare. M. S. M. and P. F. J. conceived the present project together and collaborated on the design of the data analyses and preparation of the manuscript. M. S. M. carried out the data analyses and summarised the results in tables and figures. Any opinions, findings, conclusion or recommendations expressed in the 
present publication are those of the authors and do not necessarily reflect the view of the United States Department of Agriculture.

\section{References}

1. Kuta I, Parizkova J \& Dycka J (1970) Muscle strength and lean body mass in old men of different physical activity. J Appl Physiol 29, 168-171.

2. Koopman R (2011) Dietary protein and exercise training in ageing. Proc Nutr Soc 70, 104-113.

3. Rosenberg IH (1997) Sarcopenia: origins and clinical relevance. J Nutr 127, Suppl. 5, 990S-991S.

4. Rosenberg IH (1989) Summary comments. Am J Clin Nutr 50, $1231-1233$.

5. Visser M \& Schaap LA (2011) Consequences of sarcopenia. Clin Geriatr Med 27, 387-399.

6. Wolfe RR (2006) The underappreciated role of muscle in health and disease. Am J Clin Nutr 84, 475-482.

7. Lauretani F, Russo CR, Bandinelli S, et al. (2003) Age-associated changes in skeletal muscles and their effect on mobility: an operational diagnosis of sarcopenia. J Appl Physiol 95, 1851-1860.

8. Seguin R \& Nelson ME (2003) The benefits of strength training for older adults. Am J Prev Med 25, 3 Suppl. 2, 141-149.

9. Fiatarone MA, Marks EC, Ryan ND, et al. (1990) High-intensity strength training in nonagenarians. Effects on skeletal muscle. JAMA 263, 3029-3034.

10. Nelson ME, Fiatarone MA, Morganti CM, et al. (1994) Effects of high-intensity strength training on multiple risk factors for osteoporotic fractures. A randomized controlled trial. JAMA 272, 1909-1914.

11. Sipila S \& Suominen H (1995) Effects of strength and endurance training on thigh and leg muscle mass and composition in elderly women. J Appl Physiol 78, 334-340.

12. Evans WJ (2004) Protein nutrition, exercise and aging. J Am Coll Nutr 23, Suppl. 6, 601S-609S.

13. Koopman R \& van Loon LJ (2009) Aging, exercise, and muscle protein metabolism. J Appl Physiol 106, 2040-2048.

14. Biolo G, Tipton KD, Klein S, et al. (1997) An abundant supply of amino acids enhances the metabolic effect of exercise on muscle protein. Am J Physiol 273, E122-E129.

15. Phillips SM, Hartman JW \& Wilkinson SB (2005) Dietary protein to support anabolism with resistance exercise in young men. J Am Coll Nutr 24, 134S-139S.

16. Houston DK, Nicklas BJ, Ding J, et al. (2008) Dietary protein intake is associated with lean mass change in older, community-dwelling adults: the Health, Aging, and Body Composition (Health ABC) Study. Am J Clin Nutr 87, 150-155.

17. Beasley JM, LaCroix AZ, Neuhouser ML, et al. (2010) Protein intake and incident frailty in the Women's Health Initiative observational study. J Am Geriatr Soc 58, 1063-1071.

18. Starling RD, Ades PA \& Poehlman ET (1999) Physical activity, protein intake, and appendicular skeletal muscle mass in older men. Am J Clin Nutr 70, 91-96.

19. Baumgartner RN, Waters DL, Gallagher D, et al. (1999) Predictors of skeletal muscle mass in elderly men and women. Mech Ageing Dev 107, 123-136.

20. Haub MD, Wells AM, Tarnopolsky MA, et al. (2002) Effect of protein source on resistive-training-induced changes in body composition and muscle size in older men. Am J Clin Nutr 76, 511-517.

21. Campbell Ww, Barton ML Jr, Cyr-Campbell D, et al. (1999) Effects of an omnivorous diet compared with a lactoovovegetarian diet on resistance-training-induced changes in body composition and skeletal muscle in older men. Am J Clin Nutr 70, 1032-1039.

22. Glass D \& Roubenoff $\mathrm{R}$ (2010) Recent advances in the biology and therapy of muscle wasting. Ann N Y Acad Sci 1211, 25-36.

23. National Center for Health Statistics (2002) National Health and Nutrition Examination Survey. General Documentation. http://www.cdc.gov/nchs/data/nhanes/gendoc.pdf (updated June 2002; cited 13 November 2008).

24. Centers for Disease Control and Prevention (CDC), National Center for Health Statistics (NCHS) (2011) National Health and Nutrition Examination Survey Data. Hyattsville, MD: US Department of Health and Human Services. http:// www.cdc.gov/nchs/nhanes/nhanes_questionnaires.htm (updated 18 November 2011; cited 5 March 2012).

25. Centers for Disease Control and Prevention (CDC), National Center for Health Statistics (NCHS) (2006) National Health and Nutrition Examination Survey. Analytic and Reporting Guidelines. Hyattsville, MD: US Department of Health and Human Services. http://www.cdc.gov/nchs/data/nhanes/ nhanes_03_04/nhanes_analytic_guidelines_dec_2005.pdf (updated September 2006; cited 21 April 2011)

26. Berger MJ \& Doherty TJ (2010) Sarcopenia: prevalence, mechanisms, and functional consequences. Interdiscip Top Gerontol 37, 94-114.

27. Waters DL, Baumgartner RN, Garry PJ, et al. (2010) Advantages of dietary, exercise-related, and therapeutic interventions to prevent and treat sarcopenia in adult patients: an update. Clin Interv Aging 5, 259-270.

28. Janssen I \& Ross R (2005) Linking age-related changes in skeletal muscle mass and composition with metabolism and disease. J Nutr Health Aging 9, 408-419.

29. US Environmental Protection Agency, Office of Pesticide Programs (2011) What We Eat in America (WWEIA) Food Commodity Intake Database (FCID) 2003-2006. College Park, MD: University of Maryland.

30. Dwyer J, Picciano MF \& Raiten DJ (2003) Estimation of usual intakes: What We Eat in America-NHANES. J Nutr 133, 6095-623S.

31. National Cancer Institute (2008) Usual Dietary Intakes: SAS Macros for Running the NCI Method. National Cancer Institute. http://riskfactor.cancer.gov/diet/usualintakes/macros.htm (updated 15 February 2008; cited 19 February 2008).

32. Centers for Disease Control and Prevention (CDC), National Center for Health Statistics (NCHS) (2005) National Health and Nutrition Examination Survey. Physical Activity Questionnaire. Hyattsville, MD: US Department of Health and Human Services. http://www.cdc.gov/nchs/data/nhanes/ nhanes_03_04/mi_paq_c.pdf (updated September 2005; cited 23 June 2010).

33. Willett WC, Howe GR \& Kushi LH (1997) Adjustment for total energy intake in epidemiologic studies. Am J Clin Nutr $\mathbf{6 5}$, Suppl. 4, 1220S-1228S (discussion 9S-31S).

34. Davis CG \& Biing-Hwan L (2005) Factors Affecting US Beef Consumption. Economic Research Service, United States Department of Agriculture. http://www.ers.usda.gov/ publications/ldp/Oct05/ldpm13502/ldpm13502.pdf (updated October 2005; cited 1 February 2011).

35. Ahmed N, Mandel R \& Fain MJ (2007) Frailty: an emerging geriatric syndrome. Am J Med 120, 748-753.

36. Nair KS (1995) Muscle protein turnover: methodological issues and the effect of aging. J Gerontol A Biol Sci Med Sci 50, Special no., 107-112.

37. Peterson MD, Sen A \& Gordon PM (2011) Influence of resistance exercise on lean body mass in aging adults: a meta-analysis. Med Sci Sports Exerc 43, 249-258. 
38. Institute of Medicine of the National Academies (2002) Dietary Reference Intakes for Energy, Carbohydrate, Fiber, Fat, Fatty acids, Cholesterol, Protein, and Amino Acids (Macronutrients). Washington, DC: National Academies Press.

39. Position of the American Dietetic Association, Dietitians of Canada, and the American College of Sports Medicine (2000) Nutrition and athletic performance. J Am Diet Assoc 100, 1543-1556.

40. Iglay HB, Apolzan JW, Gerrard DE, et al. (2009) Moderately increased protein intake predominately from egg sources does not influence whole body, regional, or muscle composition responses to resistance training in older people. J Nutr Health Aging 13, 108-114.

41. Liu CK \& Fielding RA (2011) Exercise as an intervention for frailty. Clin Geriatr Med 27, 101-110.

42. Sugawara J, Miyachi M, Moreau KL, et al. (2002) Age-related reductions in appendicular skeletal muscle mass: association with habitual aerobic exercise status. Clin Physiol Funct Imaging 22, 169-172.

43. Stenholm S, Harris TB, Rantanen T, et al. (2008) Sarcopenic obesity: definition, cause and consequences. Curr Opin Clin Nutr Metab Care 11, 693-700.

\section{Appendix}

The following equations were derived for men and women to estimate the appendicular skeletal muscle mass (kg):

$$
\begin{aligned}
\text { Men }: & 41.09+(-0.02 \times \text { age })+(0.62 \\
& \times \text { arm circumference }(\mathrm{cm}))+(0.46 \\
& \times \text { calf circumference }(\mathrm{cm}))+(-0.14 \\
& \times \text { triceps skinfold thickness }(\mathrm{mm}))+(1.94 \times \text { race }) \\
& +(17.79 \times \text { height }(\mathrm{m})),
\end{aligned}
$$

Women : $24 \cdot 91+(-0 \cdot 01 \times$ age $)+(0 \cdot 21$

$$
\begin{aligned}
& \times \text { arm circumference }(\mathrm{cm}))+(0.3 \\
& \times \text { calf circumference }(\mathrm{cm}))+(0 \cdot 4 \\
& \times \text { thigh circumference }(\mathrm{cm}))+(1.8 \times \text { race })+(13.49 \\
& \times \text { height }(\mathrm{m})),
\end{aligned}
$$

where race was a dummy variable coded 1 for nonHispanic black and 0 for all other racial-ethnic categories. 